\title{
Associação de megabacteriose, aspergilose e candidíase em periquitos australianos (Melopsittacus undulatus) em cativeiro, Marília, SP: relato de caso
}

\section{Association megabacteriosis, aspergillosis and candidiasis in australian parakeet (Melopsittacus undulatus) in captivity, Marilia, SP: case report}

\author{
Tarcísio Macedo Silva, ${ }^{* *}$ Adriano Sakai Okamoto, ${ }^{* * *}$ Bruna Domeneghetti Smaniotto, ${ }^{* *}$ Leonardo Fabrício Pavan, ${ }^{* * * *}$ \\ Raphael Lúcio Andreatti Filho***
}

\begin{abstract}
Resumo
Infecções fúngicas causadas por Macrorhabdus ornithogaster, Aspergillus flavus, Candida albicans tem sido descritas em várias espécies aviárias sendo responsáveis por promover alterações gastrointestinais, respiratórias, neurológicas e dermatológicas entre outras. O presente artigo descreve aspectos clínicos, anatomopatológicos, procedimentos diagnósticos, medidas preventivas e terapêuticas de um caso crônico de três infecções fúngicas concomitantes em periquitos australianos (Melopsittacus undulatus) provenientes de um criatório comercial encaminhado ao laboratório de Ornitopatologia do Hospital Veterinário da Faculdade de Medicina Veterinária e Zootecnia da Universidade Estadual Paulista (FMVZ - UNESP), campus Botucatu - São Paulo, Brasil.
\end{abstract}

Palavras-Chave: Aspergillus flavus, Macrorhabdus ornithogaster, Candida albicans, fungo, psitacídeos.

\begin{abstract} (FMVZ - UNESP), Botucatu campus - São Paulo, Brazil.

\section{Introdução}

A presença de fungos no meio ambiente como saprófitas, bem como a exposição das aves a estes micro-organismos em seu habitat normal, associadas a falhas nutricionais, estresse, debilidade, imunossupressão, antibioticoterapia extensiva, falta de saneamento, entre outros fatores, constituem situações favoráveis à proliferação destes patógenos causando enfermidades micóticas em aves (Kunkle, 2003; Dahlhausen, 2006).

Entre as principais enfermidades fúngicas que acometem aves domésticas criadas em cativeiro destacam-se as provocadas por fungos dos gêneros: Aspergillus, Criyptococus, Candida e Macrorhabdus. Outras raras micoses são causadas por agentes dos gêneros: Microsporum, Penicillium, Nocardia, Rhinosporidium e Mucor (Coles 2007; Dahlhausen, 2006).
\end{abstract}

Fungal infections caused by Macrorhabdus ornithogaster, Aspergillus flavus, Candida albicans has been described as responsible for promoting gastrointestinal, respiratory, neurological and dermatological disorders in several avian species. The present paper describes the clinical signs, histopathological exams, treatment and preventions and of a chronic case of three fungal infections concomitant in australian parakeet (Melopsittacus undulatus). The animals came from a commercial breeding facility and sent to Ornitopathology Laboratory of the Veterinary Hospital, Veterinary Medicine and Animal Science Faculty, Paulista State University

Keywords: Aspergillus flavus, Macrorhabdus ornithogaster, Candida albicans, fungus, pscittacidae.

Megabacteriose é o termo empregado para denominar infecções gástricas causadas pelo micro-organismo Macrorhabdus ornithogaster (Van Herck et al., 1984), fungo ascomiceto, anamórfico (Tomaszewski et al., 2003) que coloniza o istmo, proventrículo e o ventrículo (Van Herck et al., 1984) de várias espécies de aves, sendo comum em periquitos (Melopsittacus undulatus) (Baker, 1985; Gerlach, 2001). É visto como um componente normal da microbiota ou como agente causal de uma condição patológica aguda ou crônica, relacionada com lesões inflamatórias leves e gastrites hemorrágicas em proventrículo, além de sinais de disfagia, regurgitação e vômito, diarreia, anorexia e mortalidade (Moore et al., 2001, Phalen, 2006).

Micoses causadas por fungos do gênero Aspergillus spp. são relativamente comuns em aves e mamíferos (Tell, 2005), sendo Aspergillus fumigatus, seguido por Aspergillus flavus, as

*Recebido em 16 de outubro de 2013 e aceito em 23 de junho de 2014.

${ }^{*}$ M.V. Residentes. UNESP - Faculdade de Medicina Veterinária e Zootecnia, Departamento de Clínica Veterinária, Laboratório de Ornitopatologia Botucatu-SP.

***Docentes. UNESP - Faculdade de Medicina Veterinária e Zootecnia, Departamento de Clínica Veterinária, Laboratório de Ornitopatologia Botucatu-SP.

****M.V. Residente. UNESP - Faculdade de Medicina Veterinária e Zootecnia, Departamento de Clínica Veterinária, Laboratório de Patologia Veterinária Botucatu-SP.

Autor para correspondência: sakai@fmvz.unesp.br 
principais espécies patogênicas isoladas em aves diagnosticadas com aspergilose (Keymer, 1982). Tal fato deve-se à presença desses agentes no meio ambiente como micro-organismos ubíquos, saprófitas e oportunistas (Beernaert, et al., 2010; Jones e Orosz, 2000), e à exposição das aves aos esporos que, quando inalados, são capazes de promover severas alterações clínicas, incidindo principalmente em infecções relacionadas com o trato respiratório inferior (Dahlhausen, 2006). Os sinais clínicos e as lesões patológicas dependem da forma apresentada pela enfermidade. O modo agudo é resultante da inalação de grande quantidade de esporos e a crônica relaciona-se com a imunossupressão. Observa-se em pássaros: letargia, inapetência, anorexia, rinite, sinusite, traqueíte, mudanças na vocalização, aerossaculite, dispneia, dermatites, ascite, problemas oculares, sinais neurológicos entre outros (Beernaert et al., 2010; Dahlhausen, 2006; Tell, 2005).

Visto como micro-organismo normal da microbiota intestinal de aves, ou como um agente oportunista de distribuição mundial, Candida spp., principalmente a espécie Candida albicans, provoca micoses endógenas que acometem principalmente o trato digestivo superior das aves (Kunkle, 2003). Em geral psitacídeos são as principais espécies acometidas por candidíases (Velasco, 2000), apresentando-se deprimidos, com esvaziamento gástrico retardado, regurgitação, inapetência e dificuldade na digestão dos alimentos (Dahlhausen, 2006).

O diagnóstico precoce dessas infecções consiste em um desafio para tratamento e controle (Dahlhausen, 2006). A anamnese, o histórico, a avaliação clínica do paciente e exames radiográficos são fundamentais ao diagnóstico. Porém, a confirmação só é possível por meio de histopatologia, testes envolvendo DNA, testes sorológicos, cultura, coloração de Gram entre outros testes (Beernaert et al., 2010; Coles 2007; Gerlach, 200; Jones e Orosz, 2000; Velasco, 2000). Medidas preventivas incluem a eliminação de fatores predisponentes, controle ambiental, diminuição do $\mathrm{pH}$ da água e correção da dieta o tratamento dessas se dá por meio de terapêutica com utilização de antifúngicos (Dahlhausen, 2006).

Devido aos poucos relatos sobre a ocorrência associada de enfermidades fúngicas, o objetivo do presente artigo foi descrever aspectos clínicos e anatomopatológicos, procedimentos diagnósticos, medidas preventivas e terapêuticas de um caso crônico e associado de três infecções fúngicas em periquitos australianos.

\section{Relato de caso}

Foram encaminhados ao serviço de Ornitopatologia do Hospital Veterinário da FMVZ/UNESP - Botucatu para realização de atendimento clínico e exame necroscópico, 21 filhotes de periquitos australianos (Melopsittacus undulatus) machos e fêmeas, sendo três vivos e 18 mortos, provenientes de um criatório comercial localizado na cidade de Marília-SP.

O criador relatou que há aproximadamente dois anos vem tendo problemas com elevada morbidade e mortalidade dos filhotes com até 10 dias de vida. De um total de aproximadamente 50 filhotes, chegando as taxas de morbidade e mortalidade a $100 \%$ e $90 \%$ respectivamente no último mês. Relatou ainda que os animais apresentavam paresia de membros inferiores e superiores, alterações respiratórias, diarreia, emagrecimento progressivo, disfagia e problemas com empenamento. As aves adultas eram mantidas em viveiros, com cerca de 40 indivíduos em cada viveiro, e os filhotes, em ninhos de madeira dentro dos viveiros. Tanto as aves adultas quanto os filhotes se alimentavam de sementes de painço e almeirão.

Os animais foram tratados pelo proprietário repetidamente, de modo frustrado e sem orientações prévias, com cloridrato de oxitetraciclina, sulfonamida e enrofloxacina. Durante o exame físico observou-se dificuldade respiratória marcada pela respiração com o bico aberto, incoordenação motora (asas caídas e movimentos involuntários das patas e cabeça), apatia, sonolência, presença de fezes secas aderidas ao redor da cloaca, desidratação, dilatação abdominal e regurgitação.

As três aves vivas foram eutanasiadas por meio de deslocamento cervical, segundo a resolução 1000 de 11 de maio de 2012 (CFMV, 2012). Todas as 21 aves foram necropsiadas. Durante a necropsia observaram-se dilatação do inglúvio, associada à inflamação do órgão, dilatação proventricular com presença de muco e pontos de hemorragia, presença de sementes não digeridas no intestino delgado, hemorragia pulmonar, ascite e encefalite. Alguns aspectos relatados estão expostos na Figura 1.

Fragmentos de inglúvio e pulmão foram coletados e cultivados em ágar dextrose Sabouraud e incubados sob uma temperatura de $37^{\circ} \mathrm{C}$. Após 48 horas de incubação, o cultivo apresentou o crescimento de colônias leveduriformes de coloração esbranquiçada, aspecto pastoso e superfície irregular (Figura 2C). Microscopicamente as colônias leveduriformes apresentaram pseudohifas compatíveis com Candida spp. Realizou-se prova do tubo germinativo para detecção da patogenicidade, seguindo procedimentos descritos por Andreatti Filho (2009) identificando Candida albicans como mostrado na Figura 2A.

No cultivo dos pulmões, observou-se o crescimento de colônias filamentosas de cor verde amarelada com bordos de coloração branca (Figura 2B), identificadas microscopicamente como Aspergillus flavus. Para diagnosticar a presença de Macrorhabdus ornithogaster, foram confeccionados esfregaços das mucosas de proventrículos corados pela coloração de Gram, além de histopatologia do órgão (Figura 3A).

O exame histopatológico do proventrículo revelou a presença do micro-organismo Macrorhabdus ornithogaster infiltrado nos tecidos e na superfície da mucosa, além de grande quantidade de heterófilos e plasmócitos em meio às células epiteliais e no lúmen do órgão, caracterizando um processo inflamatório crônico ativo, presença de áreas de necrose nas vilosidades e vacuolização de células epiteliais (Figura 3B).

Foi instituído o tratamento antifúngico à base de cetoconazol (200mg por litro de água de bebida) durante 30 dias, acidificação da água com vinagre de maçã, correção dietética devido à má alimentação (Anderson, 1993; Beernaert et al., 2010; Moore et al., 2001). Como protocolo periódico de profilaxia e desinfecção ambiental dos viveiros e ninhos recomendou-se a remoção da sujeira, utilização de vassoura de fogo, lavagem com água e sabão e desinfecção com Biofor® na concentração de $20 \mathrm{~mL}$ do produto para cada 10 litros de água. 


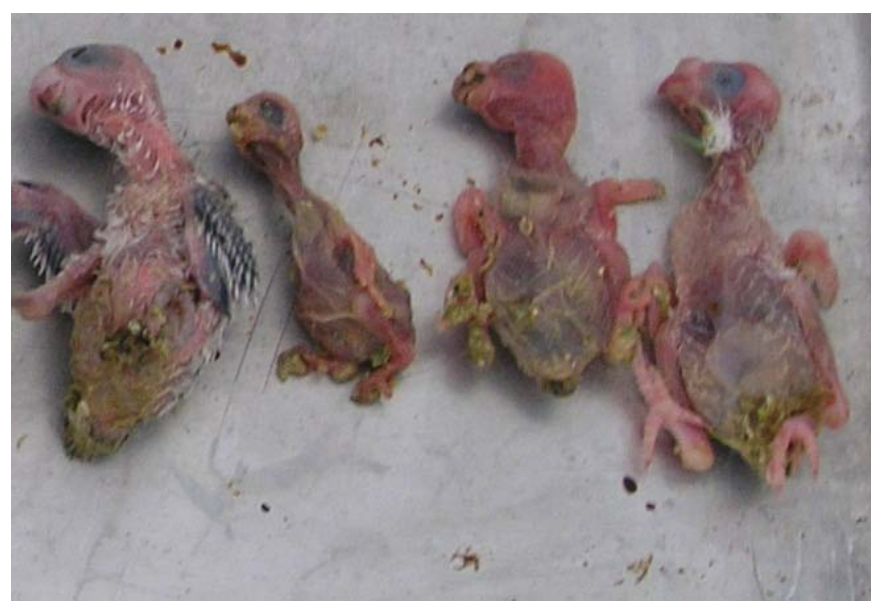

Figura 1: (Melopsittacus undulatus) presença de fezes aderidas ao redor da cloaca, desidratação, dilatação do inglúvio e dilatação abdominal

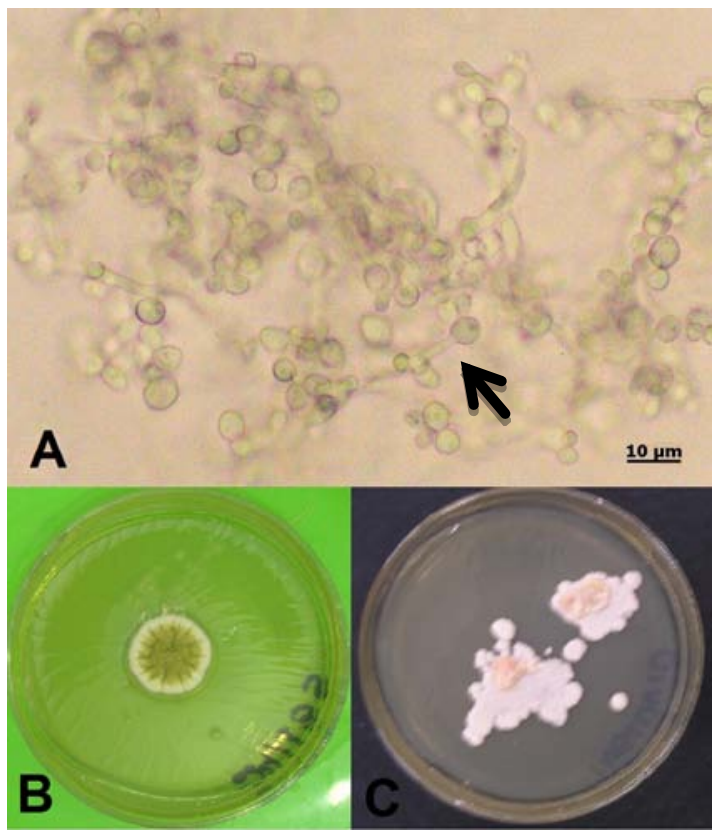

Figura 2 A: Candida albicans prova do tubo germinativo. B: Colônia de Aspergillus flavus em cultivo micológico de pulmão de periquito australiano. C: Colônias de Candida albicans em cultivo micológico de inglúvio de periquito australiano

\section{Discussão}

Em psitacídeos, a ocorrência de uma ou duas infecções fúngicas tem sido descrita em: Melopsittacus undulatus (Filippich e Hendrikz, 1998), Amazona aestiva (Carrasco et al., 1998), Cacatua sulphurea sulphurea (Anderson, 1993), Nymphicus hollandicus (Vasconcelos et al., 2011), Agapornis fischeri (Nouri e Kamyabi, 2010). Não foram descritos ainda no Brasil relatos da ocorrência associada de aspergilose, megabacteriose e candidíase em Melopsittacus undulatus.

A associação de falhas nutricionais, estresse, debilidade, imunossupressão, diminuição de ventilação, mudanças de temperatura, falhas de higienização, ambiente inapropriado, antibioticoterapia prolongada, administração de corticoides, traumatismos, predispõem ao surgimento de infecções fúngicas em aves (Dahlhausen, 2006; Tell, 2005). As alterações gastrintestinais, respiratórias, neurológicas e dermatológicas encontradas nas aves deste estudo foram descritas por Beernaert et al. (2010), Dahlhausen (2006), Moore et al. (2001), Phalen (2005) e Velasco, (2000) e nos permitiu inferir se tratar de infecções fúngicas por Macrorhabdus ornithogaster, Candida spp. e Aspergillus spp. Essas informações, associadas aos achados anatomopatológicos, histopatológicos, ao isolamento e à identificação dos agentes foram fundamentais para concluir o diagnóstico das infecções, sendo possível relacionar o quadro em questão e os fatores predisponentes à ocorrência das micoses e a um caso crônico das enfermidades.

O histórico de alta morbidade e mortalidade de filhotes reforça a ideia de que indivíduos mais jovens são mais susceptíveis aos agentes, e que as aves adultas apresentam certa resistência às micoses. A presença de Candida albicans e Aspergillus flavus e outros fungos no ambiente atuando como patógenos oportunistas (Jones e Orosz, 2000) requer grande esforço e a implantação de medidas sanitárias objetivando diminuir o número de leveduras e esporos nesse ambiente, de modo a evitar recontaminações.

O histórico de medicação sem orientação veterinária e ausência de sucesso à terapia antimicrobiana subsidiou o diagnóstico, servindo também como um alerta, para a adoção das boas práticas terapêuticas, principalmente quando se trata de medicações para aves individuais ou de criações. Todo medicamento pode causar efeitos adversos, quando utilizado de forma inadequada, podendo provocar agravação do processo patológico (Fraga et al., 2011).

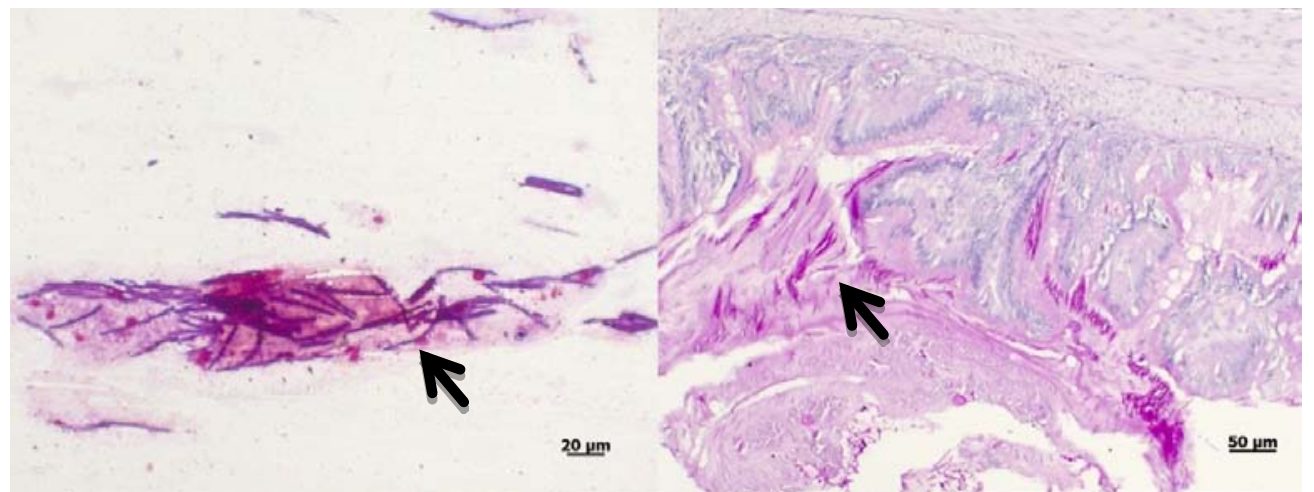

Figura 3 A: Esfregaço de proventrículo de periquitos australianos (Melopsittacus undulatus) evidenciando a presença de Macrorhabdus ornithogaster (Gram). B: Corte histológico de proventrículo, presença de Macrorhabdus ornithogaster infiltrada nos tecidos (PAS).
A terapia antifúngica com cetoconazol associada à correção dietética, diminuição do $\mathrm{pH}$ da água e adoção de medidas sanitárias resultou em melhora clínica dos animais, além de uma diminuição nos quadros de morbidade e mortalidade dos filhotes a 10 e $5 \%$ respectivamente. 


\section{Conclusões}

Com base nos achados clínicos, anatomopatológicos e laboratoriais, firmou-se o diagnóstico de megabacteriose, aspergilose e candidíase concomitantes. De acordo com as informações bibliográficas disponíveis, esse parece ser o primeiro caso

\section{Referências}

ANDERSON, N. L. Candida/Megabacteria proventriculitis in a lesser sulphur-crested cockatoo (Cacatuasulphurea sulphurea). Journal Association of Avian Veterinary, v. 7, n. 4, p. 197-201, 1993.

ANDREATTI FILHO, R. L. Enfermidades micóticas. In: BERCHIERI JUNIOR, A.; SILVA. E. M.; DI FÁBIO, J.; SESTI L.; ZUANAZE, M. A. F. Doenças das aves. 2. ed. Campinas: FACTA, 2009, p.805-818.

BAKER, J. R. Clinical and pathological aspects of "going light" in exhibition budgerigars (Melopsittacus undulatus). Veterinary Record, v. 116, p. 406-408, 1985.

BEERNAERT, L. A.; PASMANS, F.; VAN WAEYENBERGHE.; HAESEBROUCK, F.; MARTEL, A. Aspergillus infections in birds: a review. Avian Pathology, v. 39, n. 5, p. 325-331, 2010.

CARRASCO, L.; GÓMEZ-VILLAMANDOS, J. C.; JENSEN, $\mathrm{H}$. E. Systemic candidiasis and concomitant aspergillosis and zygomycosis in two amazon parakeets (Amazona aestiva). Mycoses, v. 41, p. 297-301, 1998.

CFMV. Conselho Federal de Medicina Veterinária Resolução ${ }^{\circ}$ 1000 , de 11 de maio de 2012, que dispõe sobre procedimentos e métodos de eutanásia em animais e dá outras providências. Disponível em: <http://www.cfmv.org.br/portal/legislacao/ resolucoes/resolucao_1000.pdf>. Acesso em: $10 \mathrm{fev}$ 2014.

COLES, B. Essentials of avian medicine and surgery. 3. ed. Oxford: Blackwell Publishing, 2007, 406 p.

DAHLHAUSEN, R. D. Implication of mycoses in clinical disorders. In: HARRISON, G. J.; LIGHTFOOT, T. (Eds.). Clinical Avian Medicine. Spix Publishing: Palm Beach, 2006, p. 691704.

FILIPPICH, L.J.; HENDRIKZ, J.K. Prevalence of megabacteria in budgerigar colonies. Australian Veterinary Journal, v. 76, p. 92-95, 1998.

FRAGA, M. E.; MEDEIROS, M. E. S.; NEVES, D. M. Estudo de Aspergilli durante o período de quarentena de psitacídeos do Centro de Triagem de Animais Silvestres (CETAS) IBAMA, Seropédica, $\mathrm{Rj}^{*}$. Revista Brasileira de Medicina Veterinária, v. 33, n. 2. p. 68-72, 2011.

GERLACH, H. Megabacteriosis. Seminars in Avian and Exotic Pet Medicine, v. 10, p. 12-19, 2001.

JONES, M. P.; ORORZ, S. P. The diagnosis of aspergillosis in birds. Seminars in Avian and Exotic Pet Medicine, v. 9, p. 52-58, 2000. associado dessas enfermidades em periquitos australianos descrito no Brasil. Salientamos que a implementação de um tratamento correto e a aplicação de medidas sanitárias são importantes para melhora no quadro clínico dessas enfermidades, e para a diminuição nos quadros de mortalidade de filhotes.

KEYMER, I. F. Mycoses. In: PETRAK, M.L. Diseases of Cage and Aviary Birds. 2. ed. Philadelphia: Lea \& Fabiger, p. 599-605. 1982.

KUNKLE R.A. Fungal infections. In: SAIF Y.M.; BARNES H. J.; GLISSON, J. R.; FADLY A.M.; MCDOUGALD L.R.; SWAYNE D.E. (Eds.). Diseases of Poultry. 11. ed. Ames: lowa State Press, 2003, p. 883-902.

MOORE, R. P.; SNOWDEN, K. F.; PHALEN, D. N. A method of preventing transmission of so-called "megabacteria" in budgerigars (Melopsittacus undulatus)Journal of Avian Medicine and Surgery, v. 15, n. 4, p. 283-287, 2001.

NOURI, J.; KAMYABI, Z. Occurrence of Ventricular Candidiasis in a Lovebird (Agapornis fischeri) Iranian Journal of Veterinary Science and Technology, v.2, n. 1, p. 51-56, 2010.

PHALEN, D Diagnosis and management of Macrorhabdus ornithogaste $r$ (formerly Megabacteria). Veterinary Clinics of North America: Exotic Animal Practice, v. 8, p. 299-306, 2005. PHALEN, D. N. Implications of Macrorhabdus ornithogaster in clinical disorders. In: HARRISON, G. J, Lightfoot T (Eds.). Clinical Avian Medicine.v.2, Palm Beach: Spix Pubishing Inc, 2006, p. 705-710.

TELL, L.A. Aspergillosis in mammals and birds: impact on veterinary medicine. Medical Mycology Supplement, v. 43, supl 1, p. S7-S73, 2005.

TOMASZEWSKI, E.K.; LOGAN, K.S.; SNOWDEN, K.F.; KURTZMAN, C.P., PHALEN, D.N. Phylogenetic analysis identifies the 'megabacterium' of birds as a novel anamorphic ascomycetous yeast, Macrorhabdus ornithogaster gen. nov., sp. nov. International Journal of Systematic and Evolutionary Microbiology, v. 53, p. 1201- 1205, 2003.

VAN HERCK, H.; DUIJSER, T.; ZWART, P.; DORRESTEIN, G. M.; BUITELAAR, M.; VANDERHAGE, M. H. A bacterial proventriculitis in canaries (Serinus canaria). Avian Pathology, v. 13, p. 561-572, 1984.

VASCONCELOS, T. C. B.; LONGA, C. S.; ALBUQUERQUE, D. D. A.; COSTA, C. H. C.; BRUNO, S. F. Aspectos clínicos e anatomopatológicos de aspergilose e candidíase em calopsita (Nymphicus hollandicus): relato de caso. Revista Portuguesa de Ciências Veterinárias, v. 110, p. 109-112, 2011.

VELASCO, M. C. Candidiasis and Cryptococcosis in Birds. Seminars Avian and Exotic Pet Medicine, v. 9, n. 2, p. 75-81, 2000. 